\title{
Integrated Counselling Strategies for Children in the Substance Abused Family
}

\author{
Abijo Temitayo Serah ${ }^{1,{ }^{*}}$, Huzili $\mathrm{H}^{1}{ }^{1}$, and Hanif Suhairi $^{2}$ \\ ${ }^{1}$ Department of Education \& Sport Science, Universiti Malaysia Perlis, Perlis, Malaysia \\ ${ }^{2}$ Department of Information Technology Communication, Universiti Malaysia Perlis, Perlis, Malaysia
}

\begin{abstract}
In spite of the fact that substance abuse remains a public health challenge and social problem of enormous magnitudes, with many adolescents and families involved and affected by substance abuse disarrays the issue of proper counselling still requires more attention. In view of this therefore, this study has explored and appraised conceptually the desirability as well as applicable integrated strategies for counselling of the children in the substance abused family. Based on the appraisal of existing strategies for counseling of substance abused family then this study has been able to establish that if people involved as well as the victims of the abused will be able to learn about concepts such as inner strength, self-praise, and group praise, which may prompt setting up of goals for positive decision on their addiction. Similarly, the strategies will encourage the target family to generate more and more positive self-portraits and, as these may inspire making positive lifestyle changes.
\end{abstract}

\section{Introduction}

In families where substance are being abused, behavior becomes recurrently impulsive and communication is uncertain [1]. Family life is characterized by chaos and unpredictability. Behavior can range from loving to withdrawn to crazy. Structure and rules may be either nonexistent or inconsistent. Children, who may not understand that their parent's behavior and mood is determined by the amount of substance in their bloodstream, can feel confused and insecure. They love their parents and worry about them, and yet feel angry and hurt that their parents do not love them enough to stop using. This situation was corroborated by The American Academy of Experts in Traumatic Stress [2] who revealed that in view of the enormous challenges being faced by children in the substance abused family, many families referred to children's social care because of concerns about the children's welfare and evidence of parental substance misuse or domestic violence. More so, that parental drug abuse is a possibility factor for child abandonment and general mistreatment [3].

In spite of the fact that substance abuse remains a public health challenge and social problem of enormous magnitudes, with many adolescents and families involved and affected by substance abuse disarrays [4] the issue of proper counselling still requires more attention [5]. Similarly, Abdu-Raheem
[6] reiterated that the issue of substance abuse needs pressing responsiveness due to the startling rate and more so, that the children of these families who are innocently at risk receive less attention.

Many times, children of substance abusers are frightened. They may be the victims of physical violence or incest. They may also witness violence. As noted by NFPI Children of parents who have a drink problem often want to keep it a secret and don't feel they can invite other children home - this affects their ability to make friends and have a social life. They see other families having fun together and notice that their own family is different. Home life is tense and unpredictable. Often children don't know what to expect when they return from school or when their parent comes home after a drinking session. However, in spite of all these challenges, many of the Children of substance abused family are often not aware of how much their parents' drinking is impacting on their lives [1]. Therefore, counselling becomes imperative for children who are in this problem. Correspondingly, Kendler, Ohlsson, Sundquist and Sundquist [7] believed that the widespread prevalence of illicit substance use which is high within the family presents another indication of the need for continued research because it is a risk factor for children. Therefore, this study is set out to explore conceptually the desirability 
as well as applicable strategies for counselling of the children in the substance abused family.

\section{Prevention Strategies for substance abuse impacts on Children}

As to substance utilize and abuse, avoidance depends on previous history of conduct, and embarks to foresee and turn away planned negative outcomes from happening. Counteractive action programming as far as substance utilize and abuse is essentially conveyed to the whole school populace and frequently the entire educational system, paying little respect to students' hazard elements. More focused on aversion programs have been utilized to address the issues of students who show the hazard components sketched out in the past segment or who have a background marked by medication utilize [8]. The motivation, skills, and decision-making model of drug abuse prevention. Substance Use \& Misuse, 39, 1971-2016. Not with standing, little writing exist with respect to the school guide's part.

At the school level, two methodologies have principally been utilized to address the psychosocial advancement of students. The social impact approach [9] is utilized to improve the familiarity with natural and associate impacts which possibly advance medication utilize, create peer refusal aptitudes, and test sedate desire. The individual and social expertise upgrade approach has additionally been utilized and is viewed as more far reaching in nature [10]. These sorts of counteractive action programs show abatements of $10 \%$ to $15 \%$ in school age youngsters starting utilization of substances contrasted with students that are not part of an avoidance program. In particular, these projects both diminish in social impact and increment in individual and social abilities [8].

Prevention strategies targeting youths have evolved over more than twenty years as evaluation research has revealed more about what works [11]. Several strategies are used effectively, especially in combination (Indiana Prevention Resource Center at Indiana University):
- Information dissemination. This strategy provides awareness and knowledge of the nature and extent of alcohol, tobacco, and other drug use, abuse, and addiction and their effects on individuals, families, and communities as well as information to increase perceptions of risk. It also provides knowledge and awareness of prevention policies, programs, and services. It helps to set and reinforce norms (e.g., a policy that underage drinking and drug dealers will not be tolerated in this neighborhood).

- Prevention education. This strategy aims to affect critical life and social skills, including decision making, refusal skills, critical analysis (e.g., of media messages), and systematic and judgmental abilities.

- Alternatives. This strategy provides for the participation of targeted populations in activities that exclude alcohol, tobacco, and other drug use by youths. Constructive and healthy activities offset the attraction to, or otherwise meet the needs that are usually filled by, alcohol, tobacco, and other drug use. - Problem identification and referral. This strategy calls for identification, education, and counseling for youths who have indulged in age-inappropriate use of tobacco products or alcohol or who have indulged in the first use of illicit drugs. Activities under this strategy would include screening for tendencies toward substance abuse and referral for preventive treatment for curbing such tendencies.

- Community-based process. This strategy aims to enhance the community's ability to provide prevention and treatment services to alcohol, tobacco, and other drug use disorders more effectively. Activities include organizing, planning, enhancing efficiency and effectiveness of services implementation, interagency collaboration, coalition building, and networking. Building healthy communities encourages healthy lifestyle choices.

- Environmental approach. This strategy sets up or changes written and unwritten community standards, codes, and attitudes, influencing the incidence and prevalence of alcohol, tobacco, and other drug use problems in the general population. Included are laws to restrict availability and access, price increases, and communitywide actions. 


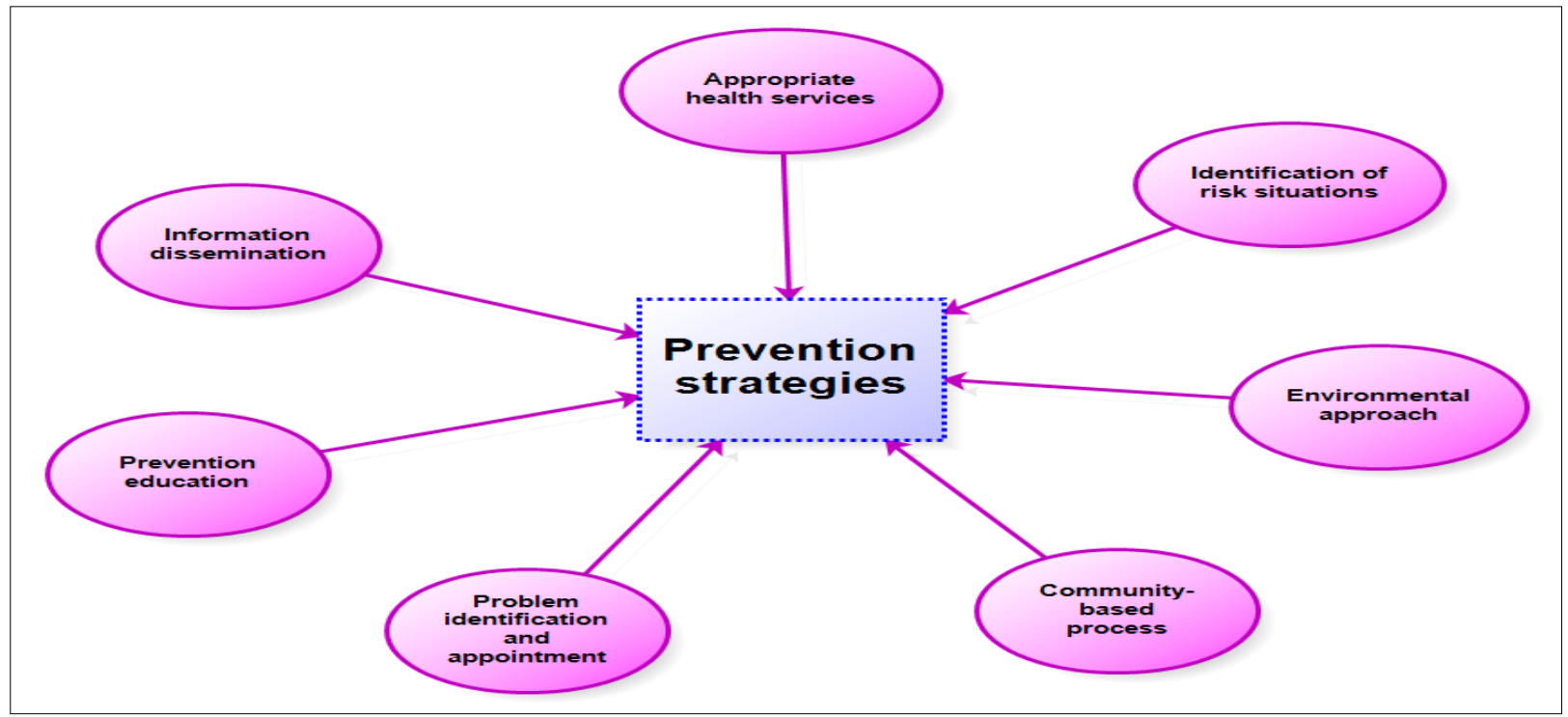

Fig. 1. Prevention strategies for substances abuse [11]

\section{Strategies for Substance abusers and Victims Counselors}

According to Igidi [12] the spate of alcohol abuse in families in Nigeria has reached alarming point because at present, only few is free of alcohol abuse and unfortunately, nothing is being done about it. Similarly, Chweya and Auya [4] lamented that in Nigeria, the situation shows there is high pervasiveness of substance use especially alcohol among family members and there is high likelihood that the frequency will continue to increase.

Therefore, in view of the fact that most of the children of substance abused family attend school, there is a tendency for them to interact with school instructors on a predictable premise in addition to that, schools are purposively arranged to give decisive counselling to such. The purposeful setup includes the school advocates' capacity to distinguish students' hazard components and hindrances to individual, social, and scholastic improvement [13].

However, in view of the increasing rate of family substance abuse [12] and the challenges and risks resulting from it [6], it thus far indicates that school counselors need to be familiar with students who may be substance abusers; students who might be at risk for becoming substance abusers; and students who have parents who have been or who are substance abusers. According to Lambie and Rokutani [14] counselors for children and students who have issues with substance abuse have three main tasks. These tasks are: identifying the signs of substance abuse; establishing a therapeutic relationship; helping the family system so that change is possible; and to be a liaison between the student, family, school, and community

\subsection{Identifying Warning Signs of Substance Abuse}

Fulfilling these functions may be challenging due to the lack of education about substance abuse that school counselors receive. School counselors and school counseling students must take it upon themselves to learn more about substance abuse disorders.

Here is a list of concerning behaviors to look for in an adolescent who might be using drugs:

$\checkmark$ Changes in school performance (falling grades, skipping school, tardiness)

$\checkmark$ Changes in peer group (hanging out with drug-using, antisocial, older friends

$\checkmark$ Breaking rules at home, school, in the community

$\checkmark$ Extreme mood swings, depression, irritability, anger, negative attitude

$\checkmark$ Sudden increases or decreases in activity level

$\checkmark \quad$ Withdrawal from the family; keeping secrets

$\checkmark$ Changes in physical appearance (weight loss, lack of cleanliness, strange smells)

$\checkmark$ Red, watery, glassy eyes or runny nose not due to allergies or cold 
$\checkmark \quad$ Changes in eating or sleeping habits

$\checkmark \quad$ Lack of motivation or interest in things other teenagers enjoy (hobbies, sports)

$\checkmark$ Lying, stealing, hiding things

$\checkmark \quad$ Using street or drug language or possession of drug paraphernalia/items

$\checkmark \quad$ Cigarette smoking

\subsection{Establishing a Therapeutic Relationship and Involving the Family}

In substance abuse intervention, treatment, and follow-up, it is important to establish a relationship in order to provide ongoing support [14]. Naturally, a school counselor has other duties to consider as part of the job, but if a school counselor is committed to counseling, then he or she can maintain a relationship with the troubled adolescent. This counseling relationship may be a source of comfort for a student struggling with substance abuse.

Involving the family is not only ethical and probably mandatory in most school districts but can also give the counselor an idea about what the student's.
Involving the family is not only ethical, and probably mandatory in most school districts, but can also give the counselor an idea about what the student's home life is like. The school counselor must consider the family system when thinking about early identification of substance abuse for two reasons: working with the parents can provide useful information about the student's substance abuse, and inviting the family to come into the school to consult with the school counselor can help the school counselor see how the family interacts [14].

\subsection{Substance Abuse Prevention and Intervention within the School}

In order to try to protect the most students possible, school counselors can advocate for substance-abuse prevention services in schools. School counselors can achieve this goal in a few ways. Counselors can use group counseling sessions to address substance-abuse issues with students who are struggling with anxiety, depression, stress, grief, or social skills. School counselors can also bring in outside agencies to provide services to individual students, targeted groups of students, or prevention programming to all students.

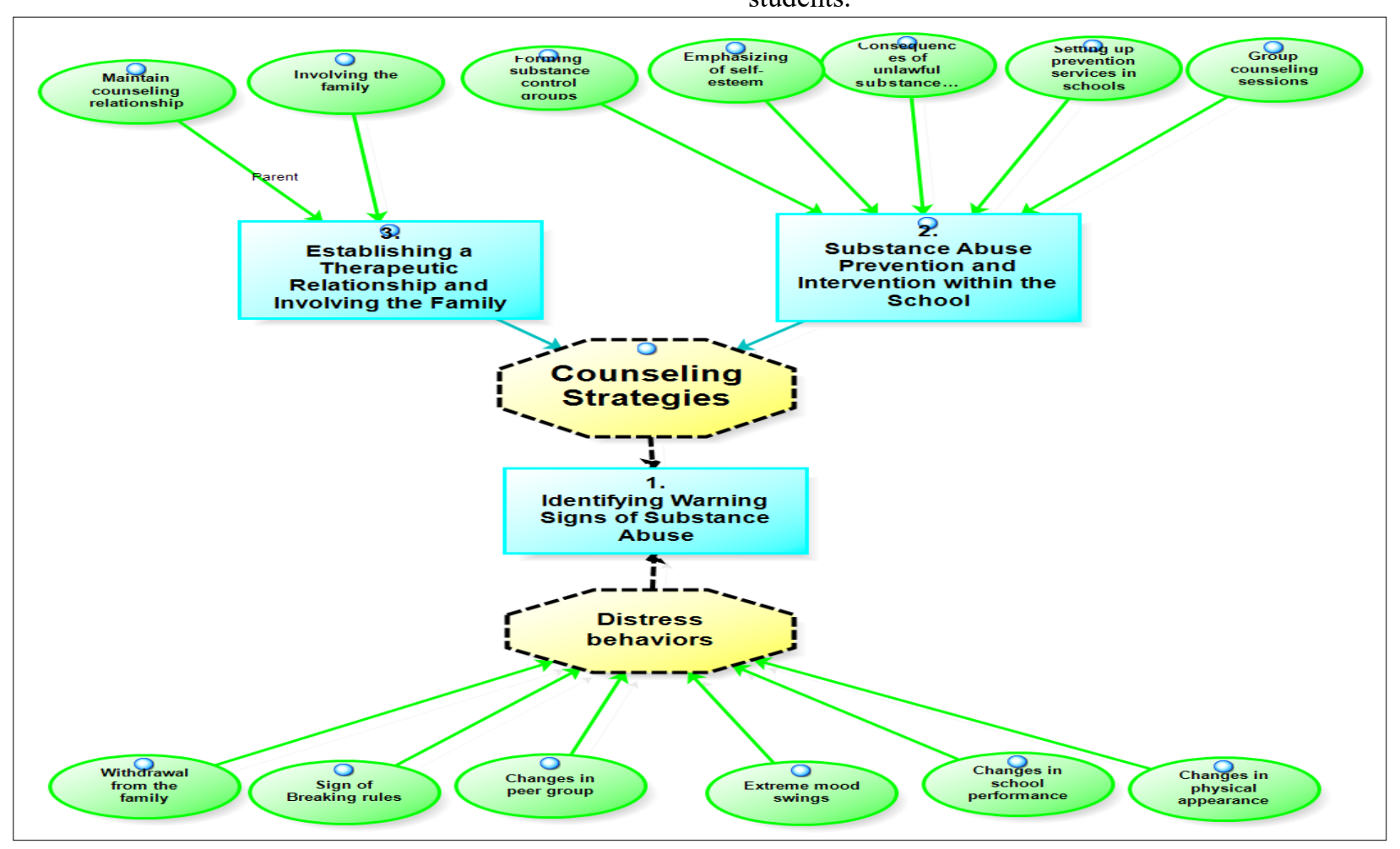

Fig. 2. Counselors Strategies for Substance Abusers and Victims [14] Source: Lambie and Rokutani (2002).

Arising from the Lambie and Rokutani [14] strategies and in view of the emerging sensitivity to issues of culture in substance abuse as revealed by DHHS [15] the need for an integrated counselling strategies for 
children of substance abuse families becomes the gaps which this study fills. The integrated strategies which are critical for both long and short-term success by setting clear and achievable goals for counselors will be a main guide for government, counselors, and agencies. Therefore, in line with UNODC, DHHS, and Baird [5, 1-16] the following basic integrated strategies are being recommended for substance counseling for substance abuse family generally.

\subsubsection{Confidentiality should be assured}

Confidentiality contributes to openness and trust which are essential to any helping relationship. If total confidentiality cannot be guaranteed, counselling staffs must make the victims aware of the possible implications of disclosure right from the beginning of the interaction. Though, due to secrecy involved, children of substance abused family may be unwilling to take up counselling opportunities when confidentiality cannot be guaranteed.

\subsubsection{Counselors should know their strengths and limitations}

Every counselor need to consider their level of skill and knowledge before intervening, identifying that restrictions which could relate to issues such as available time, confidentiality and victims acceptance.

\subsubsection{Counselling should be kept separate from discipline}

In some schools it may not be possible to have two separate staff members to provide counselling and discipline. Where possible, however, those roles should be separated in order to increase the effectiveness of intervention in both areas.

\subsubsection{De-stigmatizing without condoning drug use}

Counselors and teachers should avoid trying to force the student to change, as that may increase their resistance. In the same way, condoning or condemning the student's behaviour is counter-productive. Instead, the student should be treated as a decision-maker who has certain reasons for his or her choices based on how he or she sees the world. Teachers should direct their efforts to helping the student balance the costs and benefits of his or her decisions.

\subsubsection{Concentrating on rapport and empathy}

Drug use is a sensitive topic and one that young people are often reluctant to talk about. Some of them believe that adults will attempt to convince them to stop, criticize their behaviour or punish them. Establishing a rapport and expressing understanding is vital to a healthy helping relationship.

\subsubsection{The role of culture and cultural backgrounds}

To develop effective counseling strategies for diverse populations, the counselor must understand the role of culture and cultural backgrounds, recognize the cultural backgrounds of substance abuse family, and know enough about their culture to understand its effect on key counseling issues. This sensitivity is important at every stage of the counseling process. Meanwhile, cultural background can affect attitudes concerning such factors as proper family behavior, family hierarchy, acceptable levels of substance use, and methods of dealing with shame and guilt. Forcing families or individuals to comply with the customs of the dominant culture can create mistrust and reduce the effectiveness of therapy. 


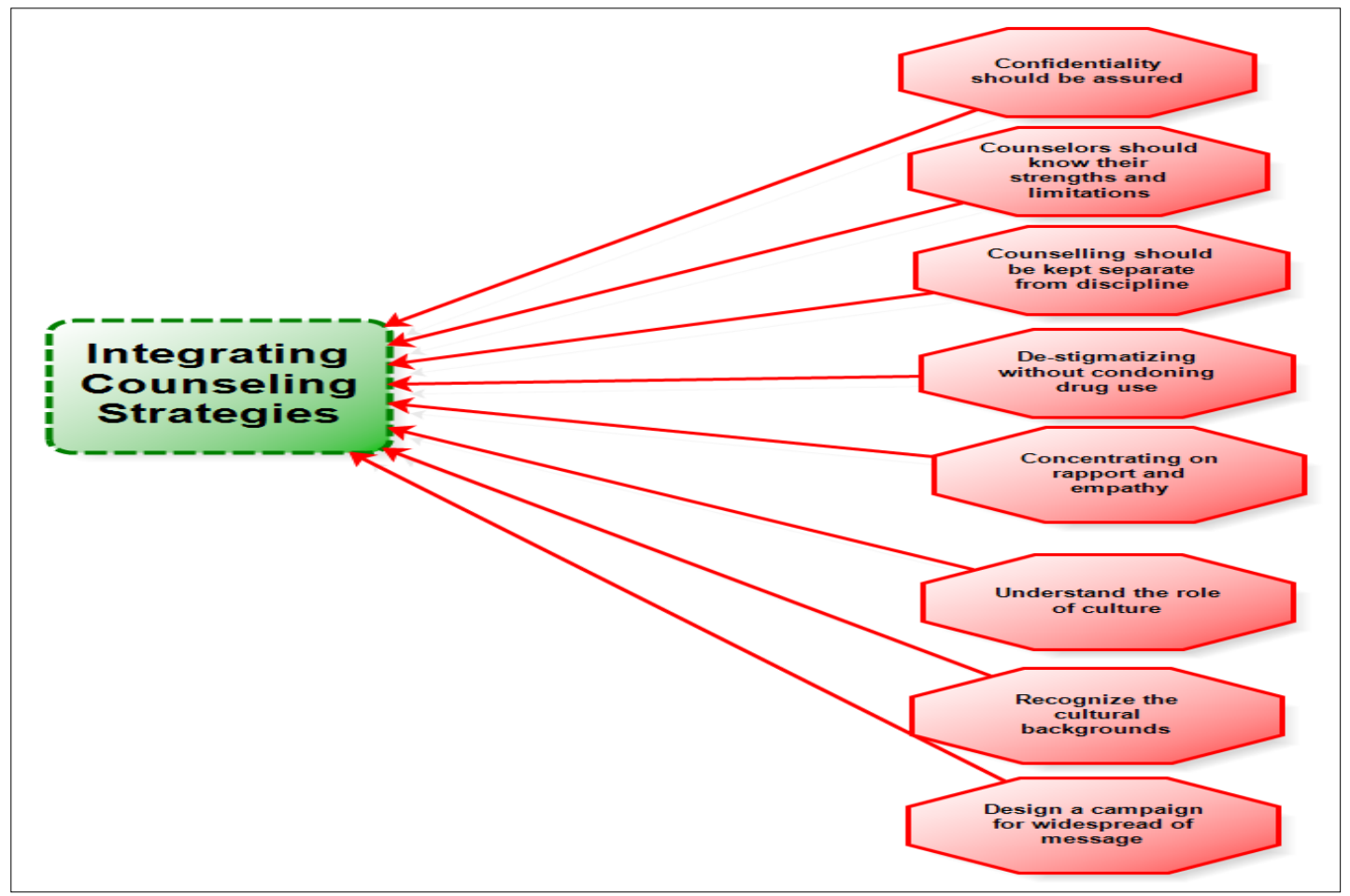

Fig.3. Integrated Counseling Strategies for substance abused families [16]

Reducing the harm to children from parental problem substance use as well as the parent themselves should become a main objective of policy and practice however much attention has not been directed at such. Therefore, this study conceptually synthesize previous strategies with what the handling requires in order to attain an effective counseling which will have major benefits for the innocent victims of family engrossed in substance abuse.

Similarly, as part of the integration, counselor should institute and sustain a campaign that will achieve widespread, frequent, and prolonged exposure to implications of substance abuse as well as the benefits derivable from the acceptance of message.

\section{Conclusion}

Based on the appraisal of existing strategies for counseling of substance abused family this study has been able to establish that if people involved as well as the victims of the abused will be able to learn about concepts such as inner strength, self-praise, and group praise, which may prompt setting up of goals for positive decision on their addiction. Similarly, the strategies will encourage the target family to generate more and more positive self-portraits and, as these may inspire making positive lifestyle changes.

As new demonstration and innovation projects continue to be implemented, expanded, and evaluated, the field continues to learn more about promising and effective approaches to holistically address the complex needs of families with substance use issues, there is need for a systematic counselling section for all the victims and their families. In particular, there is a continuing call for and movement toward enhanced collaboration among child welfare, substance abuse treatment, courts, and other systems to provide coordinated and comprehensive services of counselling to both children and their parents. Generally according to previous studies, children of parents who engage in substance abuse remained more likely to be having indication of educational difficulties, emotional and behavioral problems as well as other challenges. 


\section{References}

1. Turning Point, (2016). Bottling it up the effects of alcohol misuse on children, parents and families. Retrieved 27 March, 2016 from http://www.substancesandalcohol.ie/6276/1/ 3499-3720.pdf.

2. The American Academy of Experts in Traumatic Stress (2014). Effects of Parental Substance Abuse on Children and Families. Retrieved October 6, 2016 from http://www.aaets.org/article230.htm.

3. M. F. Taylor, D. Coall, R. Marquis, R. Batten, Int J Ment Health Addiction (2016) DOI 10.1007/s11469-016-9645-7.

4. M. Chweya, S. Auya, International Journal of innovation and scientific research, 9(1), 35-39 (2014)

5. Baird, Educational Specialist 33 (2011)

6. B. O. Abdu-Raheem, Contemporary Issues in Education Research 6(2), 233-240

7. K. S. Kendler, H. Ohlsson, K. Sundquist, J. Sundquist, JAMA psychiatry 70(2), 235-242 (2013)

8. S. Sussman, M. Earleywine, T. Wills, C. Cody, T. Biglan, C. W. DentSubstance Use \& Misuse 39, 1971-2016 (2004)

9. R. I. Evans, Preventive Medicine 5, 122-127 (1976)

10. G. J. Botvin, E. M. Botvin, Psychological Reports 70, 171-178 (1992)

11. H. Abadinsky, Drug use and abuse: A Comprehensive Introduction (USA: Wadsworth, 2011)

12. T. Igidi, (February 25, 2016). Few families are substance free in Nigeria. Daily Trust Newspaper. Retrieved February 27, 2016 from http://www.dailytrust.com.ng/news/homefront/-few-families-are-substance-free-innigeria/135355.html.

13. M. J. Ratts, A. M. Hutchins, Journal of Counseling \& Development 87, 269-275 (2009)

14. G. W. Lambie, L. J. Rokutani, A systems approach to substance abuse Counseling 5(5), 353-359 (2002)

15. DHHS (2005). Subtance Substance Abuse Treatment and Family Therapy. U.S. Department of Health And Human Services, USA: Center for Substance Abuse Treatment.
16. UNODC (2004). Nigerian Country office. Directory of treatment and rehabilitation centers. Nigeria. 